\title{
A SOLUTION
}

An Exemple as requested in the problem of $R$. Thom, $D 4 c$

by F. Takens

Consider in the plane $\mathbb{R}^{2}$ with euclidean metric $d s^{2}=d x^{2}+d y^{2}$ and unit sphere $x^{2}+y^{2}=1$ the polynomial function $f=x\left(2 x^{2}+3 t y^{2}\right), t>0$, with covectorfield $d f=3\left[\left(2 x^{2}+t y^{2}\right) d x+2 t x y d y\right]$. The set of periods at which df is linearly dependent on $a\left(x^{2}+y^{2}\right)=2[x d x+y d y]$ is given by $\left(2 x^{2}+t y^{2}\right) y-2 t x y \cdot x=$ $y\left[t y^{2}-2(t-1) x^{2}\right]=0$. Then it is easily seen that the set of points that converge to $(0,0)$ when following the gradientline of $f$ downwards is:

for $0<t \leqq 1$, the half line $\{(x, y): x \geq 0, y=0\}$

and for $t>1$, the solid angle $\left\{(x, y): x \geq 0, t y^{2}-2(t-1) x^{2} \leq 0\right\}$.

With the substitution $X=x, Y=\sqrt{t} y$ we can conclude:

The set of points in the $(X, Y)$-plane that converge to $(0,0)$ downwards when following the gradient lines of the function $x\left(2 x^{2}+3 y^{2}\right)$ with respect to the euclidean metric $d s^{2}=t d x^{2}+d y^{2}$ with unit sphere $t X^{2}+y^{2}=1$, is:

for $0<t \leq 1$, the half line $\{(X, Y): X \geqq 0, Y=0\}$, and for $t>1$, the solid angle $\left\{(X, Y): X \geq 0, Y^{2}-2(t-1) X^{2} \leq 0\right\}$. Hence the topological type of this set varies with the metric depending on $t$. 